Rev. Est. de Políticas Públicas, 5(1): diciembre 2018 - junio 2019, 1-13

http://dx.doi.org/10.5354/0719-6296.2019.50904

ISSN edición web: 0719-6296

CCopyright 2019: Universidad de Chile, Santiago (Chile)

\title{
De-Generadas: La Violencia Institucional Capacitista hacia Mujeres con Discapacidad en Chile*
}

\author{
Juan Andrés Pino-Morán y Pía Rodríguez-Garrido \\ Universidad Autónoma de Barcelona y Universidad de Barcelona
}

\section{Resumen}

El artículo tiene por objetivo problematizar la situación de las mujeres con discapacidad en el Estado de Chile, visibilizando la ausencia de la perspectiva de género en las políticas públicas sobre la discapacidad, lo cual sostiene el problema y sus representaciones históricas dominantes. Como contraparte, emerge el activismo y la agencia de las mujeres con discapacidad subvirtiendo esta situación de precariedad y avanzando en la incidencia pública nacional.

Palabras clave: Género, políticas públicas, discapacidad, exclusión social.

De-Generated: Institutional Violence Capacity towards Women's with Disabilities in Chile

\section{Abstract}

The article aims to problematize the situation of women with disabilities in the State of Chile, making visible the absence of a gender approach in public policies on disability, which supports the dominant representations of the problem. As a counterpart, the activism and agency of women with disabilities emerges to subvert the situation of invisibility and advance the national public incidence.

Keywords: Gender, public policies, disability, social exclusion.

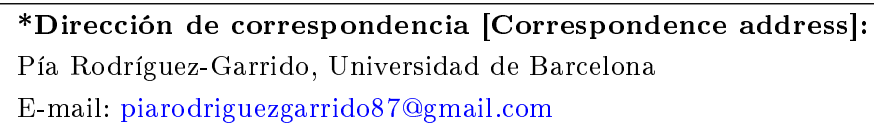

\footnotetext{
* Agradecemos a la Comisión Nacional Científica y Tecnológica de Chile (CONICYT) folio $n^{0}$ : 5489-2016. Y al grupo LIS "Estudios sociales y de género sobre la corporalidad, la subjetividad y el sufrimiento evitable" del Departamento de Sociología de la Universidad Autónoma de Barcelona
} 


\section{Introducción ${ }^{1}$}

Una sociedad heteropatriarcal, colonial, capitalista (De Sousa-Santos, 2017) y capacitista, establece patrones y mandatos normativos sobre los cuales se conducen las formas de gobierno y subjetividad. A partir de esto, se puede establecer qué cuerpos son legítimos y cuáles son ilegítimos. Por lo tanto, bajo este panorama, se piensa problematizar la posición de las mujeres con discapacidad ${ }^{2}$ en el Estado de Chile.

Ante esto, es importante destacar que las personas con discapacidad han pasado de ser un foco específico de intervención del Estado, a un trabajo gradual en la agenda pública nacional e internacional sobre desarrollo humano y capital social. Sin embargo, su materialización en políticas concretas e impacto en las vidas de las personas, aún es incipiente y precario. Esta situación se agrava cuando se piensa en clave de género ${ }^{3} \mathrm{y}$, particularmente, en las mujeres con discapacidad que viven en Chile.

Por otra parte, los estudios de la discapacidad (Morris, 1996; Barton, 1998, 2008; Ferreira, 2008, 2010; Arnau, 2017) no han tenido un impacto significativo en Chile. Existen iniciales investigaciones interdisciplinares que no han podido subvertir la inteligibilidad del fenómeno asociado a una tragedia y a un problema médico individual. Asimismo, desde los estudios de género, no ha existido una mayor preocupación por reflexionar sobre la situación de las mujeres con discapacidad como sujetas políticas y, del mismo modo, los estudios de políticas públicas no han tenido un mayor interés en la materia, quedando el debate en un plano de abandono en el país.

Por el contrario, este trabajo pretende visibilizar

\footnotetext{
${ }^{1} \mathrm{El}$ texto es parte de los análisis que se siguen en una investigación sobre activismo sociopolítico de las personas con discapacidad en Chile, en donde un foco particular de trabajo se asocia a la opresión que viven las mujeres en situación de discapacidad. Para esta ocasión, utilizaremos las referencias sobre las políticas públicas como parte del marco configuratorio de la realidad nacional.

${ }^{2}$ La discusión sobre la terminología apropiada para referirse a las mujeres que presentan condiciones y características no normativas no está saldada, sobre todo desde los movimientos sociales y queremos advertirlo. Sin embargo, en el marco de este trabajo sobre políticas públicas y género, utilizaremos la nomenclatura de la Convención Internacional de los Derechos de las Personas con Discapacidad y la utilizada por la legislación chilena vigente (Ley 20.422). A pesar de ello, utilizaremos otras terminologías, cuando por cuestiones estrictamente teóricas, se requiera.

${ }^{3}$ Para efectos de este documento, nos referiremos a perspectiva de género como sinónimo de enfoque de género, terminología que explicaremos más adelante.
}

esta situación y poner en cuestionamiento las respuestas y estrategias planteadas por el Estado de Chile. Para ello, se siguió una estructura metodológica guiada desde una reflexión teórica sustentada en un análisis documental, que permitió aproximarnos a una lectura crítica de las políticas públicas de la discapacidad como fuentes de información para describir en parte el devenir de las trayectorias de las mujeres con discapacidad en el contexto de Chile.

Se entiende el análisis documental como un conjunto de operaciones encaminadas a representar un documento y su contenido bajo una forma diferente de su forma original (Pinto-Molina, 1991). Es decir, comprender el mensaje más allá de sus significaciones primeras. En ese sentido, buscamos identificar las omisiones y sus subtextos para la elaboración de un nuevo documento que haga posible una recuperación y reparación de aquellos ámbitos que no aparecen aún en el contenido oficial (Bardin, 1986).

Siguiendo este marco analítico, pretendemos responder a las siguientes preguntas: ¿Por qué las políticas públicas sobre discapacidad en Chile no están abordadas desde una perspectiva de género?, ¿por qué es importante el enfoque de género en materia de mujeres con discapacidad?, ¿qué perspectivas se están trabajando para visibilizar esos cuerpos y trayectorias?

Para responder a estas preguntas y presentar los antecedentes de las políticas públicas de la discapacidad, se describe el panorama global en el que se configura el problema y sus soluciones. Para ello, se analizan los ejes fundamentales en los cuales se inscribe este cuadro político, jurídico y social de las personas en situación de discapacidad, específicamente, abordando la situación de las mujeres con discapacidad. Posteriormente, se describirá el contexto de las políticas públicas en Chile, problematizando el lugar que ocupa el "género", en tanto construcción cultural que se infiltra en el diseño de estas políticas públicas. Para finalizar, presentaremos la emergencia del activismo de las mujeres con discapacidad como contraparte a las propuestas del Estado y como una expresión ciudadana que pretende subvertir la situación actual e histórica del colectivo.

\section{Situando el panorama mundial de las personas con discapacidad}

Se estima que aproximadamente un $15 \%$ de la población mundial vive con algún tipo de discapacidad, es decir, más de 1.000.000.000 de personas. Cifras 
que van en alza considerando que en 1970 la población con discapacidad a nivel mundial bordeaba el $10 \%$ (Organización Mundial de la Salud, 2011).

La Organización Mundial de la Salud (OMS) refiere que entre 110 millones $(2,2 \%)$ y 190 millones $(3,8 \%)$ de personas mayores de 15 años tienen dificultades importantes para funcionar (Organización Mundial de la Salud, 2018). De acuerdo al Banco Mundial, es más probable que las personas con discapacidad experimenten situaciones socioeconómicas adversas relacionadas con la educación, peores condiciones de salud, altas tasas de desempleo y tasas más elevadas de pobreza asociadas a su condición que una persona sin discapacidad (Banco Mundial, 2017).

En ese sentido, la Encuesta Mundial de Salud realizada entre los años 2002-2004, refiere que la prevalencia de la discapacidad es mayor en países de ingresos bajos que en los países de ingresos elevados y afecta en mayor medida a las personas pobres, mujeres, ancianos y grupos étnicos (Organización Panamericana de la Salud, 2013).

La elevada incidencia registrada se asocia principalmente al envejecimiento de la población junto al aumento de las enfermedades crónicas asociadas a la discapacidad. Así también, a las condiciones ambientales, la sobrecarga de trabajo, la precarización de las ciudades, los accidentes de tránsito y el aumento de la violencia de género (Organización Mundial de la Salud, 2011).

De acuerdo a lo anterior, la OMS considera que la discapacidad es un problema global de salud pública, una cuestión de derechos humanos y una prioridad para el desarrollo mundial (Organización Mundial de la Salud, 2013).

\section{Situación mundial de las mujeres con discapacidad}

De acuerdo al Informe Mundial de Discapacidad, el índice de prevalencia de discapacidad femenina es del 19,2\%, mientras que el de los hombres es del $12 \%$ (OMS, 2011). La Comisión Económica para América Latina y el Caribe (CEPAL) indicó que entre los años 2000 y 2011 la prevalencia de discapacidad fue superior en las mujeres que en los hombres (Comisión Económica para América Latina y el Caribe, 2011: 13).

Se estima que cerca de un $60 \%$ del total de personas con discapacidad son mujeres y niñas (Cisternas,
2014). Ello podría obedecer al hecho de que la mayor esperanza de vida de las mujeres aumenta la posibilidad de tener una discapacidad. De igual modo, las mujeres que viven en situación de discapacidad en América Latina, están en un contexto de mayor vulnerabilidad económica, lo que potencia aún más el riesgo de que cualquier dificultad impacte en su desarrollo y funcionamiento eficaz en el entorno, debido a la falta de recursos que les impide costear servicios de apoyo y las ayudas técnicas necesarias (Carrillo y Molina, 2016).

En ese sentido, las mujeres con discapacidad se enfrentan a mayores dificultades, las cuales las inhabilita como sujetas autónomas dentro de la sociedad. La Organización de las Naciones Unidas (ONU) refiere la dificultad por parte de las mujeres con discapacidad en el acceso a una vivienda adecuada, a los servicios de salud, educación, formación profesional y empleo, junto a desigualdades en las contrataciones, tasas de ascensos, remuneraciones por igual trabajo y la poca participación en la toma de decisiones económicas (Organización de las Naciones Unidas, 2018).

En los últimos años, el Comité de los Derechos de las Personas con Discapacidad, organismo compuesto por expertos en la materia quienes supervisan la aplicación de la Convención sobre los Derechos de las Personas con Discapacidad (Organización de Naciones Unidas, 2009), han planteado a los Estados Partes miembros de la Convención, recomendaciones en torno a situaciones que afectan a niñas y mujeres con discapacidad, haciendo mención a diversos ámbitos de trabajo, entre ellos: la autonomía, la esterilización forzada, la discriminación y la violencia de género. En ese plano, el Comité decidió dedicar un debate general a los derechos de las mujeres y niñas con discapacidad para abordar asuntos relevantes como la interseccionalidad del género y la discapacidad, la violencia contra las niñas y mujeres con discapacidad y sus derechos sexuales y reproductivos (Organización de Naciones Unidas, 2014).

Ante esta situación, se hace necesario y urgente impulsar medidas y políticas públicas que permitan reparar y resolver el problema de las personas con discapacidad, y especialmente, atender a las necesidades especificas de las mujeres con discapacidad, que puedan acompañar el proceso de su desarrollo integral e inclusión social. 


\section{Políticas públicas para las perso- nas con discapacidad en Chile}

Entenderemos por políticas públicas como "todo lo que los gobiernos deciden hacer o no hacer" (Dye, 2008: 1). O como acción o inacción gubernamental en respuesta a problemas públicos (Kraft y Furlong, 2006). Por tanto, las políticas públicas reflejan la tensión entre lo que ha sido acordado/reconocido versus lo negado/invisibilizado para los asuntos públicos. En lo que respecta a este trabajo analítico, se contextualizará en la relevancia del enfoque de género.

Desde esa óptica, el enfoque de derechos viene a ser un marco teórico-ético desde donde problematizar las brechas entre lo discursivo y lo efectivo, observable en la materialización de políticas públicas, permitiendo identificar variables políticas, sociales, económicas y culturales que inciden en el diseño de políticas. El enfoque de derechos tiene por objetivo asegurar el cumplimiento efectivo de los derechos fundamentales, siendo utilizado en la gestión pública y como herramienta de lucha para la ciudadanía, particularmente, para los sectores y colectivos que se encuentran en desventaja (Fortunatti, 2013).

En nuestro caso, nos ayuda a pensar las relaciones de poder en la configuración de los derechos de las personas con discapacidad y, especialmente, la posición de las mujeres como imperativo para los Estados (Flores, 2013). Que tal como lo refiere Abramovich (2006), no son personas con necesidades especiales, sino sujetos de derechos, con agencias para demandar al Estado.

En materia de discapacidad en Chile, las políticas públicas están basadas en el marco de Derechos Humanos, que fue materializado a través de la Convención Internacional de los Derechos de las Personas con Discapacidad ${ }^{4}$ celebrada en el año 2006 y ratificada por Chile en julio del año 2008 (Organización

\footnotetext{
${ }^{4}$ Se reconoce como antecedente preliminar la promulgación en 1994 de la Ley № 19.284 que Establece Normas Para la Plena Integración Social de Personas con Discapacidad que crea el fondo nacional de la discapacidad (FONADIS). Luego a nivel regional, se redacta la Convención Interamericana para la Eliminación de Todas las Formas de Discriminación contra las Personas con Discapacidad de la Organización de los Estados Americanos (Organización de los Estados Americanos, 2009). Ambos antecedentes jurídicos se destacan como una primera etapa que implicó políticas públicas sobre discapacidad en Chile bajo un paradigma integrador. A partir de ese momento y a la fecha, se han dictado a lo menos 21 cuerpos normativos que han abordado directamente la temática de la discapacidad en diferentes ámbitos, donde podemos ver un avance hacia un paradigma que pretende ser más "inclusivo".
}

de Naciones Unidas, 2016), ambas instancias inscritas en un modelo social de la discapacidad (Palacios, 2008) y que impulsan la materialización de los derechos civiles y políticos, y los derechos económicos, sociales y culturales para las personas con discapacidad. Este enfoque sitúa en el plano de los derechos las diferentes esferas de la vida de las personas con discapacidad, haciendo hincapié en la posición particular que ocupan las mujeres y niñas con discapacidad en el mundo.

En este sentido, adscribiéndose a las solicitudes de la Convención, Chile fue el primer país de la región en recoger estadísticas que reflejaran la situación de las personas con discapacidad. Posteriormente, en el año 2010 y en el marco de la Ley N.ํㅜ 20.422 que establece las normas sobre igualidad de oportunidades e inclusión social de personas con discapacidad, se crea el Servicio Nacional de la Discapacidad (SENADIS) (Ley № 20.422, art.61).

Este organismo es responsable de promover y articular las políticas públicas en materia de discapacidad en todo el territorio nacional y fue el encargado de impulsar la elaboración del II Estudio Nacional de la Discapacidad (ENDISC) ${ }^{5}$, realizado en el año 2015. Esta última versión identificó que el 16,7\% de la población nacional mayor a 2 años se encuentra en situación de discapacidad, estadística que incorpora a 2.836.818 de individuos, mostrando que del total de personas adultas que presentan una situación de discapacidad leve, moderada o severa, un $24,9 \%$ son mujeres en contraste a un $14,8 \%$ de hombres en la misma condición (Servicio Nacional de la Discapacidad, 2015).

Por otra parte, en 2015 el Instituto Nacional de la Juventud (INJUV) realizó la Encuesta Nacional de Juventud que considera a la población menor de 19 años. Esta concluyó con que "las y los jóvenes que reportan mayor sintomatología depresiva, son las mujeres, quienes viven en zonas urbanas y quienes tienen entre 15 y 19 años de edad" (Instituto Nacional de la Juventud, 2015: 17), quienes podrían

\footnotetext{
${ }^{5}$ A este informe lo antecede el Primer Estudio Nacional de la Discapacidad en Chile realizado en el año 2004. Cabe destacar que existe una separación de 11 años entre cada estudio. Así también, la dimensión de discapacidad no ha sido considerada dentro de los Censos que se han realizado en el país. Esta situación ha imposibilitado conocer la realidad estadística de la población en Chile con discapacidad con mayor rigurosidad. Lo mismo sucede con las encuestas de Caracterización Socioeconómica Nacional (CASEN) quienes plantean la imposibilidad de construir un indicador de discapacidad "debido a que las preguntas relacionadas con esta materia abordan el concepto de manera parcial" (Ministerio de Desarrollo Social, 2011
} 
gatillar una situación de discapacidad.

Desde el plano de políticas públicas, está en vigencia "La Política Nacional para la Inclusión Social de las Personas con Discapacidad 2013-2020", bajo el Plan de Acción de la Política Nacional para la Inclusión Social de las Personas con Discapacidad (Servicio Nacional de la Discapacidad, 2013), que congrega las políticas públicas de todos los sectores y materializa el compromiso del Estado hacia las personas con discapacidad. Sus ejes de trabajo son salud, empleo ${ }^{6}$, educación, vivienda y cultura, entre otros.

Del mismo modo, se encuentra en vigencia el principio de "igualdad y no discriminación" desde la Constitución Política de la República chilena, que consagra en el Capítulo I, artículo $1^{\underline{0}}$, la plena igualdad de las personas en dignidad y derechos, así también en el capítulo III, artículo 19: $2^{\underline{O}}$ y $3^{\underline{O}}$ (Constitución Política de la República de Chile, 1980). Similar situación se describe en la Ley N²0.609 de julio de 2012, que establece medidas contra la discriminación, incluyendo la discapacidad como parte de la definición de discriminación arbitraria (Ley № 20.609, art. $2^{\underline{O}}$ ).

En los últimos años se han realizado actualizaciones a la propuesta nacional, ejemplo de ello es el "Plan Nacional sobre Inclusión Social de Personas en Situación Discapacidad"7, parte del trabajo de la comisión asesora presidencial, compuesta por representantes de la sociedad civil y expertos en la materia a nivel nacional e internacional (Comisión Asesora Presidencial sobre Inclusión Social de Personas en situación de Discapacidad, 2016).

A nivel local, se está impulsando como política pública la "Estrategia de Desarrollo Local Inclusivo" que tiene por objetivo fomentar el desarrollo local inclusivo a nivel comunal desde una perspectiva integral por medio del apoyo, cooperación técnica y coordinación intersectorial, para reorientar y/o profundizar las políticas inclusivas desde el desarrollo municipal (Servicio Nacional de la Discapacidad, 2017).

Sin embargo, las políticas públicas antes mencio-

\footnotetext{
${ }^{6}$ Se destaca la promulgación de la Ley № 21.015 en el año 2018, la cual incentiva la inclusión de personas con discapacidad al mundo laboral, sin embargo, no incorpora una perspectiva de género en sus normativas ni reglamentos.

${ }^{7}$ Fruto del trabajo de la Comisión Asesora Presidencial sobre Inclusión Social de Personas en Situación de Discapacidad, impulsada por el segundo mandato de la expresidenta Michelle Bachelet.
}

nadas, abordan planteamientos vinculados a las condiciones básicas de dignidad dentro de la sociedad, quedando ausente cualquier relación de estas condiciones con un enfoque de género. Ello se traduce en una invisibilización permanente por parte del Estado hacia las necesidades específicas de las mujeres en situación de discapacidad, o más bien, una clara manifestación de violencia institucional hacia esos cuerpos no normativos (Círculo Emancipador de Mujeres y Niñas con Discapacidad, 2015).

Cabe destacar la precaria agenda nacional de la discapacidad en materia de los escasos recursos económicos que le refieren. Ejemplo de ello es el presupuesto para los programas y políticas de las personas con discapacidad estipulado por el Ministerio de Desarrollo Social hacia el Servicio Nacional de la Discapacidad de este año, monto que asciende a $\$ 19.219 .270$, equivalente a un $3 \%$ aproximado de su presupuesto total (Ley $\mathrm{N}^{\circ} 20.422$ art. 61, 2018: 803). Sin embargo, aquello no justifica de ninguna manera la ausencia de ejes de trabajo en materia de género.

\section{La discapacidad desde un enfoque de género}

Como se evidencia en las estadísticas nacionales e internacionales, así como los planteamientos desde las políticas públicas de los Estados Partes -incluyendo a Chile-, existe una deuda histórica en materia de reconocer a las personas en situación de discapacidad como sujetos políticos de derecho. No obstante, esta situación se agudiza cuando pensamos en las mujeres con discapacidad, considerando que estadísticamente suponen más de la mitad de las personas con discapacidad y que se enfrentan a peores situaciones de violencia y maltrato (Organización Mundial de la Salud, 2011).

La violencia hacia las mujeres es una situación que por décadas se ha manifestado como eje central de trabajo en la agenda pública internacional, en instancias como la Convención sobre la Eliminación de todas las Formas de Discriminación contra la Mujer (CEDAW) y sus protocolos formativos, la Declaración sobre la Eliminación de la Violencia Contra la Mujer, la Convención sobre los Derechos Políticos de la Mujer, la Organización de las Naciones Unidas y su departamento ONU Mujeres, la Cuarta Conferencia Mundial sobre la Mujer en Beijing, la Comisión de la Condición Jurídica y Social de la Mujer, entre otras.

Desde Naciones Unidas y la Asamblea General del 
año 1994, definieron como violencia en contra la mujer:

\begin{abstract}
Todo acto de violencia basado en la pertenencia al sexo femenino que tenga o pueda tener como resultado un daño o sufrimiento físico, sexual o psicológico para la mujer, así como las amenazas de tales actos, la coacción o la privación arbitraria de la libertad, tanto si se producen en la vida pública como en la vida privada.
\end{abstract}

(Organización de Naciones Unidas (1994))

A partir de la problematización de las diversas manifestaciones de violencias y maltratos hacia las mujeres y, específicamente, hacia las mujeres con discapacidad, es que se plantea desde los Estados y organismos internacionales, generar políticas desde una perspectiva de género, las cuales propongan salidas a esta situación histórica. En ese contexto, se entiende la "incorporación de una perspectiva de género", de acuerdo a lo que plantea Lagarde (1996):

La perspectiva de género permite analizar y comprender las características que definen a las mujeres y a los hombres de manera específica, así como sus semejanzas y diferencias. Esta perspectiva de género analiza las posibilidades vitales de las mujeres y los hombres; el sentido de sus vidas, sus expectativas y oportunidades, las complejas y diversas relaciones sociales que se den entre ambos géneros, así como los conflictos institucionales y cotidianos que deben enfrentar a las maneras en que lo hacen [...] No ha sido sencillo lograr la aceptación de la perspectiva de género, ya que hacerlo conduce a desmontar críticamente la estructura de la concepción del mundo y de la propia subjetividad. Y no es casual que así suceda. La representación del orden genérico del mundo, los estereotipos sociales y sus normas, son fundamentales en la configuración de la subjetividad de cada quien y en la cultura.

(Lagarde, 1996: 2-3)

Este ordenamiento social nos permite analizar todo el complejo sistema sexo-género (Rubin, 1975), ya que el proceso de génerización se establece socioculturalmente en una relación dialógica entre hombres y mujeres, como dos caras de una misma moneda, pero que se expresan en relaciones de poder desiguales, por ello es necesario una traducción correcta de las tensiones que viven los géneros considerando las bases de desigualdad en las cuales se fundamentan sus dinámicas sociales (Scott, 1996).

En el caso de las mujeres con discapacidad, su propia condición las mantiene subjetivadas al vínculo de las injusticias que no permiten un bienestar en su identificación de género. Similar reflexión se realiza desde las comprensiones feministas, así Viñuela (2009) describe que uno de los encuentros más claros entre la teoría feminista y la discapacidad es lo relativo al cuerpo y sus conceptualizaciones enmarcados en la sujeción de la identidad, enfatizando en que el control histórico sobre los cuerpos es una característica común de las mujeres y de las personas con discapacidad (Viñuela, 2009).

Por ello, desde un marco institucional, Lagarde (1996) enfatiza en la importancia de la utilización del enfoque de género en escenarios corporativos, específicamente, en políticas públicas, manifestando que

de tanto usar el término en la formulación de políticas públicas y debido a las formas tecnocráticas y autoritarias de ponerlas en práctica a través de mandatos institucionales, la perspectiva de género ha sido víctima de la burocratización por parte de quienes impulsan acciones que inciden en la reorganización social, la reconversión económica y política y la aculturación de las mujeres. Sus escasos conocimientos y sus visiones que van desde el antifeminismo consciente o ignorante soterrado, hasta el explícito y agresivo, impiden una comprensión cabal de la profundidad de los planteamientos.

(Lagarde, 1996: 8)

Concordando con Lagarde, identificamos que existe una preocupación desde las organizaciones internacionales por incorporar la perspectiva de género como eje interseccional de trabajo como lo hemos mencionado anteriormente; sin embargo, no vemos un compromiso consistente, ya que todos los abordajes planteados son incipientes y restringidos, más aun cuando se reducen en su complejidad y no tienen una continuidad en políticas y planes de trabajo sustentables a largo plazo. La violencia -en todas sus manifestaciones- hacia las mujeres con discapacidad, amerita una transformación constitutiva en los marcos legislativos y políticas públicas de los Estados Parte, comprendiendo que la mujer con discapacidad está sometida a múltiples discriminaciones y/o opresiones, manifestadas en la constante invisibilización de sus derechos, a su vez atravesados por los 
ejes de opresión que encontramos en la raza, clase, etnia, edad, orientación sexual, entre otras expresiones.

En ese sentido, se plantea que el enfoque de género dominante ${ }^{8}$ tiene relación con una construcción patriarcal, capitalista, colonial y capacitista, cumpliendo a cabalidad los roles hegemónicos establecidos en este tipo de sociedad, dentro de los cuales las mujeres con discapacidad quedan en los márgenes. Por tanto, surgen las preguntas: ¿cómo incorporamos el enfoque de género en las políticas públicas para las mujeres con discapacidad? y ¿qué dificulta su materialización en los planes y programas?

Es importante deconstruir la comprensión de género desde la óptica de las mujeres con discapacidad, de lo contrario se continuará visibilizando desde parámetros normalistas atribuidos a ciertas características físicas, sensoriales y cognitivas de un modelo hegemónico y homogéneo en tanto se categoriza al género femenino. Por lo tanto, un primer desafío es realizar un análisis situado de la realidad de las mujeres con discapacidades.

Así, es necesario insistir que las problemáticas que sortean las mujeres con discapacidad en la sociedad no solo tienen las complejidades atribuidas a su condición -las cuales hemos manifestado explícitamente durante este documento-, sino también a las problemáticas propias del género femenino, expresadas de una manera imbricada. Por lo tanto, un segundo desafío, sería dimensionar la interseccionalidad en materia de discapacidad y género.

En ese sentido, Morris (1998) enfatiza en que las líneas de pensamiento feminista con mayor aplicación para los estudios de la discapacidad, son aquellas que no trabajan exclusivamente desde el enfoque de género. Aclara que hablar de doble discriminación no permite entrelazar las dos categorías (mujer y discapacidad) y analizarlas en conjunto, sino que las separa como dos ámbitos diferenciados.

Por ello la relevancia de cuidar el abordaje desde una posible "perspectiva de género", ya que frecuentemente las mujeres con discapacidad se observan desde escenarios de tragedia (Villaró y Galindo, 2012; Del Rio et al., 2013; Robbins, 2016) vinculados a las violencias más escabrosas que pueden sortear

\footnotetext{
${ }^{8}$ Realizamos esta acepción considerando que en las ocasiones que la perspectiva de género ha estado presente en las políticas públicas, se refiere siempre a mujeres preferentemente heterosexuales, urbanas, capacitadas, que se asumen con un tipo de cuerpo estándar y homogéneo y, por tanto, no visibiliza e incorpora a las mujeres con discapacidad.
}

desde su situación de discapacidad, como esterilizaciones forzosas, abortos coercitivos, maternidades desarraigadas y reiteradas agresiones sexuales. Se debe comprender que las mujeres con discapacidad no son víctimas, sino que la sociedad les ha establecido ese rol, siendo victimizadas históricamente, anulando su capacidad de agencia.

En el caso de Chile, no se contempla ningún abordaje serio en esta materia. Cuando se considera, por ejemplo, en los escasos proyectos concursables -que están fuera de la lógica de derechos- queda reducida a una dimensión cuantificable en las pautas evaluativas de los programas de SENADIS como asignación de bonificaciones en los fondos concursables. Esto se observa en el Programa de Apoyos a Estudiantes en Situación de Discapacidad en Instituciones de Educación Superior, en el Fondo Nacional de Proyectos Inclusivos y en el Concurso Nacional del Programa de Apoyo a Instituciones Educativas para la Inclusión de Estudiantes en Situación de Discapacidad.

Similar situación develamos al analizar las "articulaciones" entre el Servicio Nacional de la Mujer y la Equidad de Género (SernamEG) y el Servicio Nacional de la Discapacidad (SENADIS). Se observa la carencia en materia de profundidad y seriedad para abordar las problemáticas de las mujeres con discapacidad desde un enfoque de género. Ejemplo de ello se manifiesta en el "Plan Nacional Igualdad entre Hombres y Mujeres 2018-2030", en donde se puede apreciar la nula visibilidad en la incorporación de mujeres con discapacidad. Esto se agudiza cuando en el mismo documento refieren la participación del Estado de Chile en la Convención sobre los Derechos de las Personas con Discapacidad del año 2006.

En ese sentido, la aplicabilidad de la perspectiva de género no comprende una transversalidad real desde las políticas públicas, su materialidad en mesas de trabajo y protocolos se vislumbra superficial y fragmentada. Lo mismo se observa en la creación de la Mesa Técnica Intersectorial de Derechos Sexuales y Reproductivos de las Personas con Discapacidad, celebrada en marzo del 2017, que tiene características higienistas, normalizadoras y centralistas. En esta instancia se intenta plantear una propuesta en el marco de la obligatoriedad por parte del Estado de Chile en respuesta al Informe presentado por la Organización de las Naciones Unidas el pasado 13 de abril de 2016, en el cual se describe explícitamente la preocupación por parte del Comité "que las políticas de género y las políticas de discapacidad del Estado parte no incluyan a las mujeres con discapacidad, y 
que tampoco existan políticas y estrategias dirigidas a ellas específicamente". (Art. 6, nํ13, p.2).

Ante esto nos preguntamos ¿en qué radica esta persistente invizibilizacion del enfoque de género en las políticas públicas sobre discapacidad de Chile?, ¿cuáles son sus causas y consecuencias?

En ese plano, identificamos consistentemente una des-generación de las políticas y estrategias públicas de la discapacidad, atribuible a la constante representación histórica capacitista sobre el colectivo, que visualiza las opresiones que viven las mujeres con discapacidad por fuera de los mandatos hegemónicos de género. La categoría o el etiquetaje de la discapacidad las posiciona en una "nueva identidad", donde quedan invisibilizados los procesos de generización, y en este caso, las identificaciones de femineidad quedan anuladas en la categoría de la discapacidad. De ahí vemos la dificultad de posicionar en la agenda de políticas públicas ese sujeto (i)legítimo e (in)deseable. Un cuerpo que nadie quiere habitar, ni nombrar.

Las políticas públicas de la discapacidad en Chile, al omitir el enfoque de género, perpetúan las desigualdades y favorecen las representaciones dominantes que asimilan esa innombrable posición que puede suponer la "mujer con discapacidad". Lo cual sigue reproduciendo las desigualdades entre hombres y mujeres, sosteniendo los estereotipos de discriminación. Los escasos recursos destinados siguen favoreciendo preferentemente a los hombres, lo que permite ver el patrón heteropatriarcal capacitista de la racionalidad pública en su máxima expresión.

\section{La agencia de las mujeres con dis- capacidad en Chile}

Como expresión de resistencia y subversión ante las diferentes expresiones de gubernamentalidad (Foucault, 1996) a las que se ven enfrentadas las mujeres que discapacita el Estado de Chile, emerge la agencia como praxis sociopolítica que interpela justicia y reconocimientos en las luchas de acción colectiva por derechos e inclusión ciudadana. Estas movilizaciones surgen de una vulnerabilidad entendida -desde una perspectiva feminista Butlerianaen diálogo con agencias encarnadas que se mueven en un doble sentido de la palabra, "como movimiento del cuerpo y como movimiento político" (Butler, 2017: 140).

En Chile, la emergencia del activismo de las muje- res con discapacidad desde una perspectiva feminista se encuentra en un proceso gradual de problematización y visibilización. En este sentido destacamos el trabajo que desarrolla el Círculo Emancipador de Mujeres y Niñas con Discapacidad de Chile (CIMUNIDIS), el Observatorio de los Derechos Sexuales y Reproductivos de las Personas con Discapacidad (ODISEX) y el Colectivo: No es lo Mismo Ser Loca que Loco; las que se destacan a nivel nacional por conformar espacios autogestionados de expertas por experiencia que se enuncian desde una primera persona.

Estos ejemplos de activismos y agencias de las mujeres con discapacidad en Chile son concordantes con el desarrollo del feminismo de la discapacidad que se desarrolla a nivel internacional (Morris, 1996; Allué, 2003; Angelino, 2014), los cuales plantean que la discriminación interseccional no es una suma de discriminaciones, sino una experiencia agravante sobre la vida misma (Arnau, 2017; Gomiz, 2017).

La necesidad de una accesibilidad a las políticas públicas con enfoque de género, al espacio público y hasta el propio cuerpo, son algunas de las demandas de los colectivos de mujeres con discapacidad en Chile que tensionan los mandatos de la organización social y sus instituciones.

El activismo de las mujeres con discapacidad en Chile se puede interpretar como la contraparte del Estado. En ese sentido, CIMUNIDIS en el año 2013 presentó un Informe sombra ${ }^{9}$ a la $9^{\mathrm{a}}$ sesión del Comité de Expertos de la Convención por los Derechos de las Personas con Discapacidad, en colaboración a la Corporación Humanas y a otras 21 ONGs para el Comité de Derechos Humanos de Naciones Unidas en el examen de Chile sobre el Pacto de Derechos Civiles y Políticos en el año 2014 (Benavides, 2013), planteando las reiteradas violaciones a los derechos humanos que se ven enfrentadas las mujeres con discapacidad por parte del Estado de Chile.

Estos movimientos asociativos de mujeres son fundamentales en sus expresiones des-estigmatizadoras y liberadoras. El fortalecimiento de estas diversas agencias puede significar un canal importante que vehiculice sus reivindicaciones y luchas, posibilitando su incidencia política en la agenda pública nacional e internacional.

\footnotetext{
${ }^{9}$ Informe paralelo que presenta la sociedad civil a modo de contrastar la versión oficial que presenta el Estado de Chile a Naciones Unidas. Es una manera de participar plenamente en el proceso de seguimiento a la aplicación de la Convención Internacional de los Derechos de las Personas con Discapacidad.
} 


\section{Discusión}

La situación de precariedad en materia de género dentro de las políticas públicas de discapacidad en Chile es alarmante (Pino y Rodríguez, 2017). La violencia que las mujeres en situación de discapacidad deben sortear no solo proviene desde la sociedad, sino también desde el propio Estado chileno y sus instituciones. Así lo manifiesta el "Estudio Exploratorio de violencia contra las mujeres con discapacidad de Chile, Uruguay y Costa Rica" que reafirma la violencia ejercida por las instituciones al momento de colocar en disputa los derechos humanos de las mujeres con discapacidad. Con ello, describe la inviabilidad desde un plano legislativo en profundizar a partir de un enfoque de garantías y derechos para los casos de violencia hacia las mujeres con discapacidad (Poblete y Bozo, 2016).

Lo anterior es ratificado por los diagnósticos realizados desde las organizaciones internacionales (Organización de los Estados Americanos, 2009; para América Latina y el Caribe, 2012; Organización de las Naciones Unidas, 2018), que son claros: las problemáticas de las mujeres en situación de discapacidad siguen empapadas de violencia y discriminación, las cuales condicionan negativamente las posibilidades de desarrollo y participación social (Mun Man et al., 2006). Más aún, comprendemos que la violencia hacia las mujeres con discapacidad no tiene una causa individual, sino que es un problema multifactorial, ocasionado por un sistema patriarcal que organiza la estructura social, excluyendo y vulnerando a las mujeres. En ese sentido, la activista feminista de la discapacidad Carme Riu expresa "tal vez deberíamos hablar de personas vulneradas más que de personas vulnerables" (Freixanet et al., 2015: 233) haciendo hincapié en las estructuras sociales y estatales que producen dicha problemática.

Desde ese plano, el "Segundo Manifiesto de los Derechos de las Mujeres y Niñas con Discapacidad de la Unión Europea. Una herramienta activistas y responsables políticos" (Foro Europeo de la Discapacidad, 2011) y el "Comité Español de Representantes de Personas con Discapacidad" (Comité Español de Representantes de Personas con Discapacidad, 2013) a través del manual (volumen II) de "Transversalidad de género en las políticas públicas de discapacidad", manifiestan la necesidad de incorporar la interseccionalidad en las políticas de género y discapacidad, que permitan superar el patrón masculino que sigue constituyendo el modelo sobre el que se diseñan políticas e intervenciones públicas, manteniendo así en la invisibilidad a las mujeres y niñas.
No obstante, una situación incipiente se presenta en la Región de Aragón de España, quienes este 2018 adoptaron un nuevo protocolo para combatir la violencia por motivo de género e incluye las necesidades de las mujeres con discapacidad por primera vez, siendo la primera región de España que reconoce las desigualdades y adopta medidas para enfrentar su vulnerabilidad (Instituto Aragonés de la Mujer, 2018).

En Chile, lamentablemente se proyecta un panorama poco auspicioso para los años venideros, ya que de acuerdo a la Agenda de Género del programa actual de gobierno ${ }^{10}$, enfatizan en la elaboración de 6 objetivos y medidas con el fin de generar una sociedad desarrollada que se base en que tanto hombres y mujeres sean reconocidos como sujetos de derechos y en igualdad de condiciones, no obstante, en lo extenso de los objetivos planteados, y bajo ningún punto de vista, se aborda la situación de las mujeres con discapacidad en Chile (Ministerio de la Mujer y la Equidad de Género, 2018).

Similar situación se manifiesta en las incipientes mesas de trabajo gestionadas desde el SernamEG y SENADIS, aún carentes de profundidad en los planteamientos de estrategias desde una perspectiva de género, ya que esta última, solo se reduce a comprensiones técnicas/operativas del concepto (Servicio Nacional de la Discapacidad, 2016).

Esta conflictiva relación entre las instituciones y las mujeres con discapacidad también es problematizada recientemente desde perspectivas feministas. Estas enfatizan en las violencias de género que se someten las mujeres con discapacidad en las instituciones, producto de que estas últimas no están preparadas para atender las demandas específicas de las mujeres con discapacidad, más bien se centran en una visión homogenizante y des-generada de estas (Arnau, 2005).

Desde los estudios y análisis que vienen desarrollando los movimientos sociales y el activismo de las personas en situación de discapacidad de Chile, expresan la preocupación por la violencia de género, ejemplo de ello es la feminización de los diagnósticos psiquiátricos, en tanto su porcentaje es mayor en mujeres que en hombres; junto al espiral creciente de medicalización que tienen las mujeres en el consumo de ansiolíticos y antidepresivos, como una expresión más de desigualdad de género y discapacidad (Cea, 2017).

\footnotetext{
${ }^{10}$ Gobierno de Sebastián Piñera (2018-2022).
} 


\section{Conclusión}

A partir del análisis realizado podemos concluir que la ausencia del enfoque de género en las políticas publicas de Chile infiltra una comprensión cultural dominante que oprime a las mujeres en situación de discapacidad, lo cual representa un agravante de vulnerabilidad y precarización de sus condicionantes de vida. El Estado de Chile ejerce una violencia de género institucional capacitista patriarcal, ya que las políticas públicas omiten, privilegian, jerarquizan y ordenan la población y sus grupos bajo criterios de capacidad y normalidad: social, cultural, económica, sexual y corporal, donde los cuerpos de las mujeres con discapacidad quedan olvidados, ocupando un lugar periférico dentro de la sociedad heteropatriarcal.

Dimensionar esta invisibilización como violencia de género en las problemáticas de la discapacidad, constituye una forma de discapacitación hacia todas las mujeres. Ello no solo afecta a las mujeres con discapacidad, sino también a aquellas mujeres que mayormente ejercen cuidados de las mujeres con discapacidad, quedando de manifiesto la urgencia en la elaboración de una política pública que tenga como eje central una ética del cuidado desde una perspectiva de género.

Con esta realidad y persistencia histórica no podemos hablar de justicia sin antes avanzar en inclusión social, en un pleno reconocimiento de nuestras diferencias y singularidades, donde el análisis de género forme parte constitutiva de nuestras identificaciones y trayectorias. Para avanzar en esta materia debemos visibilizar a las mujeres con discapacidad en toda su diversidad y heterogeneidad, no solo como un grupo específico con dificultades o como víctimas de un entramado de discriminaciones, las cuales se ven expresadas en la intersección del género con la clase, la raza, la sexualidad, y la capacidad; sino más bien como parte de la riqueza humana que convive en/con la comunidad y que tienen sus propias agencias de fortalecimiento.

Para finalizar, el activismo de las mujeres con discapacidad en Chile supone un giro epistemológico y político relevante para avanzar en materia de equidad de género, participación y derechos humanos. Su visibilización y articulación en los próximos años, podría suponer modificaciones importantes, las cuales impliquen un cambio institucional, político y cultural urgente para nuestra sociedad.

\section{Referencias}

Abramovich, V. (2006). Una aproximación al enfoque de derechos en las estrategias y políticas de desarrollo. Revista Cepal, 88.

Allué, M. (2003). DisCapacitados: La reivindicación de la igualdad en la diferencia. Bellaterra, Barcelona.

Angelino, M. (2014). Mujeres intensamente habitadas: ética del cuidado y discapacidad. La Hendija, Paraná.

Arnau, S. (2005). La cara oculta de la violencia: la violencia de género contra la(s) mujer(es) con discapacidad(es). GIAT DiscapacidadFundación Isonomía Igualdad Oportunidades. Recuperado en: http://www.fekoor.com/.

Arnau, S. (2017). El modelo de asistencia sexual como derecho humano al auto-erotismo y el acceso al propio cuerpo: un nuevo desafío para la plena implementación de la filosofía de vida independiente. Intersticios. Revista Sociológica de Pensamiento Crítico, 11(1):19-37. Recuperado en: http://www.intersticios.es/.

Banco Mundial (2017). Apartado de Discapacidad. Recuperado en: http://www.bancomundial.org/.

Bardin, L. (1986). El Análisis de Contenido. Akal, Madrid.

Barton, L. (1998). Discapacidad y sociedad. Morata, Madrid.

Barton, L. (2008). Superar las barreras de la discapacidad. Morata, Madrid.

Benavides, M. (2013). Círculo Emancipador de Mujeres y Niñas con Discapacidad de Chile. Recuperado en: disabilitycouncilinternational.org/.

Butler, J. (2017). Cuerpos aliados y lucha política: hacia una teoría performativa de la asamblea. Paidós, Madrid.

Carrillo, M. y Molina, P. (2016). Inclusión social de las personas con discapacidad en las Américas. Organización de los Estados Americanos, Washington.

Cea, J. (2017). Seminario Activismo y Movimientos Sociales en Salud Mental: Hacia una despatologización de las diferencias. Santiago de Chile. 
Círculo Emancipador de Mujeres y Niñas con Discapacidad (2015). Presentación de la Relatora de Discapacidad del Alto Comisionado de Derechos Humanos de Naciones Unidas para la Convención de los Derechos de las Personas con Discapacidad [CDPD] relación a mujeres y niñas con Discapacidad. Ginebra, Suiza.

Cisternas, M. S. (2014). Evolución Internacional y cambio de paradigmas sobre las personas con discapacidad. Desafíos en el enfoque de derechos humanos para el siglo XXI. Recuperado en: http://www.upla.cl/.

Comisión Asesora Presidencial sobre Inclusión Social de Personas en situación de Discapacidad (2016). Propuesta Plan Nacional sobre Inclusión Social de Personas en situación de Discapacidad. Recuperado en: http://archivospresidenciales.archivonacional.cl/.

Comisión Económica para América Latina y el Caribe (2011). División de Población de la CEPAL. Las Personas con Discapacidad en América Latina: del reconocimiento juridico a la desigualdad real. Recuperado en: http://www.larediberoamericana.com/.

Comité Español de Representantes de Personas con Discapacidad (2013). La transversalidad de género en las políticas públicas de discapacidad-manual volumen II. Recuperado en: https://www.cermi.es/.

Constitución Política de la República de Chile (1980). Bases de la Institucionalidad, Capítulo I. Art. $1^{\circ}$. Recuperado en: https://www.camara.cl/.

Del Rio, E., Megías, J., y Exposito, F. (2013). Gender-based violence against women with visual and physical disabilities. Psicothema, volumen 25 .

Dye, T. (2008). Undestanding pubic policies. Pearson Prentice Hall, New jersey.

Ferreira, M. (2008). La construcción social de la discapacidad: habitus, estereotipos y exclusión social. Nómadas. Revista Crítica de Ciencias Sociales y Jurídicas. 17:1.

Ferreira, M. (2010). Una aproximación sociológica a la discapacidad desde el modelo social: apuntes caracterológicos. Revista Española de investigaciones sociológicas, 124:141-174.
Flores, T. (2013). Análisis y diseño de alternativas de políticas públicas con enfoque de derechos. En Ganuza, E., Aleuy, M., y Erazo, X., editores, Políticas Públicas: Exigibilidad y Realización de Derechos. LOM editores, Santiago de Chile:.

Foro Europeo de la Discapacidad (2011). $2^{\circ} \mathrm{Ma}$ nifiesto de los Derechos de las Mujeres y Niñas con Discapacidad de la Union Europea. Una herramienta para activistas y responsables politicos. Recuperado en: https://www.cermi.es/.

Fortunatti, R. (2013). El reconocimiento de derechos. En Ganuza, E., Aleuy, M., y Erazo, X., editores, Politicas Públicas: Exigibilidad y Realización de Derechos. LOM, Santiago de Chile:.

Foucault, M. (1996). Defender la Sociedad. Buenos Aires: Fondo de Cultura Económica.

Freixanet, M., Alamany, L., Caballero, I., Crosas, I., García-Santesmases, A., Pié, A., Riu, M. C., y Serres, A. (2015). Génere I Diversitat Funcional. Una Violencia Invisible. Institut de Ciéncies Politiques i Socials, Barcelona.

Gomiz, M. (2017). Visibilizar la discapacidad: hacia un modelo de ciudadanía inclusiva. Fragua, Madrid.

Instituto Aragonés de la Mujer (2018). Protocolo de Coordinación Interinstitucional para la prevención de la Violencia de Género y Atención a las víctimas en Aragón. Recuperado en: http://www.educaragon.org/.

Instituto Nacional de la Juventud (2015). Octava Encuesta Nacional de Juventud. Recuperado en: https://biblioteca.digital.gob.cl/.

Kraft, M. y Furlong, S. (2006). Public policy: politics, analysis and alternatives. CQ Press, Washington.

Lagarde, M. (1996). El Género, fragmento literal: 'La perspectiva de género' en Género y feminismo. Desarrollo humano y democracia. Horas y HORAS, Madrid.

Ley $\mathrm{N}^{\circ} 20.422$ art. 61 (2018). Inclusión Social de Personas con Discapacidad. Diario Oficial de la República de Chile. Santiago de Chile, actualizada y publicada el 23/05/2018.

Ministerio de Desarrollo Social (2011). Encuesta de Caracterización Socioeconómica Nacional. Recuperado en: http://observatorio.ministeriodesarrollosocial.gob.cl/. 
Ministerio de la Mujer y la Equidad de Género (2018). Plan nacional Igualdad entre Hombres y Mujeres 2018-2030. Recuperado en: https://www.minmujeryeg.cl/.

Morris, J. (1996). Encuentros con desconocidas. Feminismo y discapacidad. Narcea, Madrid.

Morris, J. (1998). Feminism, gender and disability. Ponencia presentada en un seminario en Sidney, Australia. Recuperado en: https://disability-studies.leeds.ac.uk/wpcontent/uploads/sites/40/library/morrisgender-and-disability.pdf.

Mun Man, G., Conde, A., y Portillo, I. (2006). Mujer, discapacidad y violencia. El rostro oculto de la desigualdad. Instituto de la Mujer, Madrid.

Organización de las Naciones Unidas (2018). Departamento de Asuntos Económicos y Sociales, División de Política Social y Desarrollo. Las mujeres y las niñas con discapacidad. Recuperado en: https://www.un.org/.

Organización de los Estados Americanos (2009). Convención Interamericana para la Eliminación de Todas las Formas de Discriminación contra las Personas con Discapacidad. Recuperado en: http://www.oas.org/.

Organización de Naciones Unidas (1994). Asamblea General. Declaración sobre la Eliminación de la Violencia contra la Mujer. Recuperado en: https://www.ohchr.org/.

Organización de Naciones Unidas (2009). Derechos Humanos. Oficina del Alto Comisionado. Comité de los Derechos de las Personas con Discapacidad. Recuperado en: https://www.ohchr.org/.

Organización de Naciones Unidas (2014). Conferencia de los Estados Partes en la Convención sobre los Derechos de las Personas con Discapacidad. Recuperado en: http://www.un.org/.

Organización de Naciones Unidas (2016). Observaciones finales sobre el informe inicial de Chile. Mujeres con discapacidad. Art. 6. Recuperado en: http://acnudh.org/.

Organización Mundial de la Salud (2011). Informe Mundial sobre la Discapacidad. Recuperado en: http://www.who.int/.
Organización Mundial de la Salud (2013). Plan de acción mundial de la OMS sobre discapacidad 2014-2021. Recuperado en: http://www.who.int/.

Organización Mundial de la Salud (2018). Discapacidad y Salud. Recuperado en: http://www.who.int/.

Organización Panamericana de la Salud (2013). Situación Mundial de la Discapacidad. Recuperado en: http://www.bvs.hn/.

Palacios, A. (2008). El modelo social de discapacidad: orígenes, caracterización y plasmación en la Convención Internacional sobre los Derechos de las Personas con Discapacidad. Cinca, Madrid.

para América Latina y el Caribe, C. E. (2012). Panorama Social de América Latina y el Caribe. Publicación de las Naciones Unidas, Santiago de Chile. Recuperado en: https://www.cepal.org/.

Pino, J. y Rodríguez, P. (2017). ¿vivir para trabajar?: Mujeres, activismo y discapacidad en chile. Intersticios. Revista Sociológica de Pensamiento Crítico, 11(2):185-198. Recuperado en: http://www.intersticios.es/.

Pinto-Molina, M. (1991). Análisis documental: fundamentos y procedimientos. Eudema, Madrid.

Poblete, S. y Bozo, N. (2016). Estudio exploratorio de violencia contra mujeres con discapacidad en tres pases de América Latina y el Caribe: Chile, Costa Rica y Uruguay. Universidad Central y Centro de Estudios Sociales y de Opinión Pública, Santiago de Chile.

Robbins, J. (2016). Violencia, género y discapacidad: La ideología de la normalidad en el cine español. Hispanofila, 177:167-178.

Rubin, G. (1975). The traffic in women: Notes on the 'political economy' of sex. En Reiter, R., editor, Toward an antropology of women. Monthly Review Press, New York.

Scott, J. W. (1996). El género: una categoría útil para el análisis histórico. En Lamas, M., editor, El Género: la construcción cultural de la diferencia sexual, pp. 265-302. PUEG, México.

Servicio Nacional de la Discapacidad (2013). Política Nacional para la Inclusión Social de las Personas con Discapacidad 2013-2020. Recuperado en: http://www.ciudadaccesible.cl/. 
Servicio Nacional de la Discapacidad (2015). Segundo Estudio Nacional de la Discapacidad. Recuperado en: http://observatorio.ministeriodesarrollosocial.gob.cl/.

Servicio Nacional de la Discapacidad (2016). Aprobación convenio marco de cooperación celebrado entre el Servicio Nacional de la Mujer y la Equidad de Género y el Servicio Nacional de la Discapacidad. Recuperado en: https://transparencia.sernam.cl/.

Servicio Nacional de la Discapacidad (2017). Estrategia de Desarrollo Local Inclusivo. Recuperado en: https://www.senadis.gob.cl/.

Viñuela, L. (2009). Mujeres con discapacidad: Un reto para la teoría feminista. Feminismo/s, 13:33-48. Recuperado en: https://www.academia.edu/.

Villaró, G. y Galindo, L. (2012). Discapacidad intelectual y violencia de género: programa integral de intervención/intelectual. Acción psicológica, 9(1):101-114. Recuperado en: http://espacio.uned.es/. 